\title{
¿Qué elegir? ¿Leche de vaca o leches vegetales?
}

\section{What to choose? Cow's milk or vegetable milks?}

\author{
Dulce E. Bautista-Soto ${ }^{a}$, Dulce A. Callejas-Escamilla ${ }^{b}$,Sandra D.J. Cortés-Morales ${ }^{c}$, Yeini De- \\ Jesús-Venancio ${ }^{d}$, Dania I. Ortiz-García ${ }^{e}$, Deyanira I. Pérez-Casasolaf, Ramirez-Moreno E ${ }^{g}$.
}

\begin{abstract}
:
Milk for human consumption comes from cattle, while those considered as vegetable milks called "vegetable drinks" are products that come mainly from oilseeds, cereals, such as oats and rice. The objective of the present work was to establish a comparison of the nutritional composition of vegetable drinks and cow's milk. The caloric, protein, lipid and saturated fatty acid content was higher in cow's milk than in vegetable drinks, while the latter have a better lipid profile due to the content of polyunsaturated fatty acids and only in the soy drink the protein intake was similar to that of cow's milk. Regarding the concentration of vitamins A and D, there was no significant difference between the two. Finally, cow's milk is a fundamental food in the diet due to the quality of its nutritional composition, but vegetable drinks can be an alternative for specific groups of the population; however, some have excess added sugars, so their consumption should be moderate.
\end{abstract}

Keywords:

Cow's milk, plant drink, nutritional information.

Resumen:

La leche de consumo humano es proveniente del ganado vacuno, mientras que las consideradas como leches vegetales denominadas "bebidas vegetales", son productos que provienen principalmente de oleaginosas, cereales, como la avena y el arroz. El objetivo del presente trabajo fue realizar una comparación de la composición nutrimental de bebidas vegetales y leches de vaca. El contenido calórico, proteico, de lípidos y de ácidos grasos saturados fue más alto en las leches de vaca que en las bebidas vegetales, mientras que estas últimas presentan un mejor perfil lipídico por el contenido de ácidos grasos poliinsaturados y solo en la bebida de soya el aporte de proteína fue similar al de la leche de vaca. Respecto a la concentración de las vitaminas A y D no hubo una diferencia significativa entre ambas. Finalmente, la leche de vaca es un alimento fundamental en la dieta por la calidad en su composición nutricional, pero las bebidas vegetales pueden ser una alternativa para grupos específicos de la población; sin embargo, algunas presentan exceso de azúcares añadidos por lo que su consumo debe ser moderado.

Palabras Clave:

Leche de vaca, bebida vegetal, información nutrimental.

\footnotetext{
${ }^{\text {a } U n i v e r s i d a d ~ A u t o ́ n o m a ~ d e l ~ E s t a d o ~ d e ~ H i d a l g o, ~ h t t p s: / / o r c i d . o r g / 0000-0003-2750-4324, ~ E m a i l: ~ d u l c e e b s @ ~ g m a i l . c o m ~}$

b Autor de correspondencia. Universidad Autónoma del Estado de Hidalgo, https://orcid.org/0000-0002-1024-9358, Email: callejasescamilladulceamor@gmail.com

c Universidad Autónoma del Estado de Hidalgo, https://orcid.org/0000-0002-4794-0409, Email: nutrisandracortes@ gmail.com

d Universidad Autónoma del Estado de Hidalgo, https://orcid.org/0000-0002-3467-114X,, Email: yeinidejesusvenancio20@gmail.com

e Universidad Autónoma del Estado de Hidalgo, https://orcid.org/0000-0002-1423-3150, Email: danniaortiz34@ gmail.com

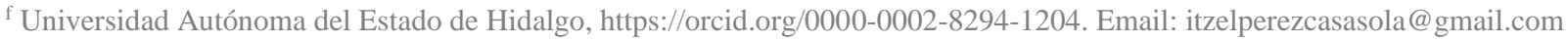

g Universidad Autónoma del Estado de Hidalgo, https://orcid.org/0000-0002-9928-8600. Email: Esther_ramirez@uaeh.edu.mx
} 


\section{Introducción}

La definición de leche está dada por su origen y hace referencia al producto de la secreción normal de la glándula mamaria de animales bovinos sanos, obtenida por uno o varios ordeños diarios, higiénicos, completos e ininterrumpidos. ${ }^{1}$ Este producto lácteo se encuentra regulado por la Norma Oficial Mexicana NOM-155-SCFI2012, la cual establece las denominaciones comerciales de los diferentes tipos de leche, que se comercializan dentro del país. Asimismo, establece que la leche para consumo humano debe ser sometida a tratamientos térmicos $u$ otros procesos que garanticen la inocuidad del producto; además puede ser sometida a operaciones tales como clarificación, homogeneización, estandarización u otras, siempre y cuando no contaminen al producto y cumpla con las especificaciones de su denominación. ${ }^{2}$

Las bebidas vegetales en donde se encuentran las denominadas comúnmente leches vegetales, son elaboradas con agua e ingredientes vegetales como cereales, leguminosas y frutos secos. En México no existe ninguna legislación que regule la producción de estos productos, por lo que en el presente trabajo serán denominadas bebidas vegetales ${ }^{3}$.De acuerdo con lo establecido por la Unión Europea el año 2017, los productos puramente vegetales no pueden comercializarse bajo las denominaciones tales como "leche", "nata", "mantequilla", "queso" o "yogur", reservadas por el Derecho de la Unión Europea a los productos de origen animal, criterio que también se aplica cuando dichas denominaciones se completan mediante menciones explicativas 0 descriptivas que indiquen el origen vegetal del producto de que se trata. ${ }^{4}$

Las principales variedades comercializadas son bebidas a base de arroz, avena, soja y almendras. Por su composición, muchas de estas bebidas presentan características particulares (sin lactosa, bajas en grasas saturadas), además muchas de ellas se enriquecen con vitaminas y minerales lo que las hacen especialmente adecuadas para ciertos sectores de la población. Muy recientemente, se ha visto un aumento en el consumo ya que son considerados como nuevos productos naturales con propiedades saludables, además de su agradable sabor que ha dado como resultado una gran aceptabilidad. ${ }^{3,5}$

\section{Metodología}

El objetivo de este estudio fue realizar una comparación de la composición nutrimental de las bebidas vegetales y leches de vaca que se expenden en supermercados de mayor importancia en la ciudad de Pachuca de Soto, Hidalgo.
Para el presente trabajo se evaluaron dos tipos de leche de origen vegetal y de vaca, seleccionando 17 diferentes marcas. Posteriormente, se revisó el etiquetado nutrimental de estos productos y su información. Los datos recopilados de la composición nutrimental fueron presentados en $100 \mathrm{~mL}$ para así poder hacer comparaciones entre los productos evaluados.

\section{Resultados y discusión}

La información nutrimental de las leches evaluadas se muestra en la tabla 1, considerando $100 \mathrm{~mL}$ de producto. Se puede observar que las diferentes marcas de leche presentan un contenido calórico entre un rango de 57 y $62.5 \mathrm{kcal}$ relacionado con el contenido de carbohidratos (4 a $5 \%$ ), grasa y proteínas (alrededor de $3 \%$ cada una). Las bebidas vegetales presentan menor contenido calórico en comparación con las de vaca por su bajo aporte en carbohidratos, proteínas y grasas, como se revisará a continuación.

Tabla 1. Contenido nutricional de leche de vaca y leches vegetales $(\mathrm{g} / 100 \mathrm{~mL})$

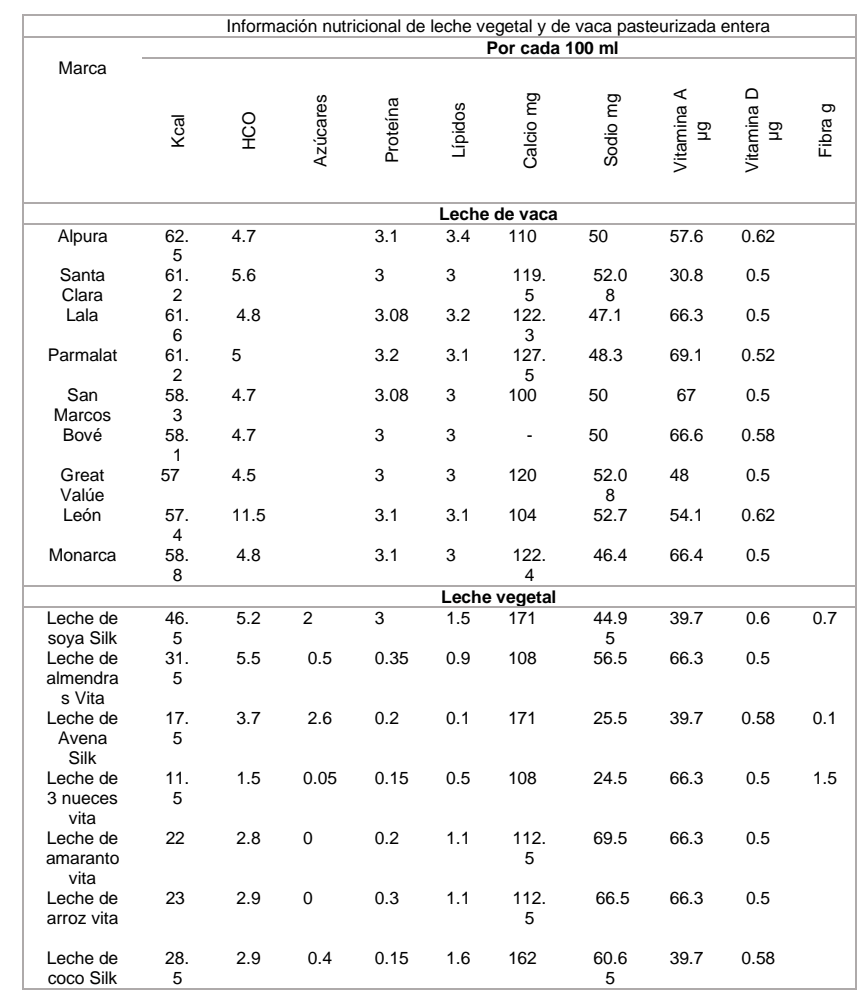

En las distintas presentaciones de leche se observó una diferencia en cuanto a los hidratos de carbono presentes, puesto que la leche de vaca contiene lactosa como hidrato de carbono mayoritario. Además de la lactosa, la leche contiene otros carbohidratos no 
absorbibles como los oligosacáridos que promueven la existencia de una flora bifidogénica en el intestino. ${ }^{6}$

El valor biológico que tienen las proteínas depende de la composición de aminoácidos, utilización fisiológica y absorción que además pudiera verse afectada por el procesamiento. En las leches de vaca se presenta un alto contenido de proteínas $(3 \mathrm{~g} / 100 \mathrm{~mL})$ caracterizada por presentar una buena fuente de aminoácidos a partir de caseína y lactoalbúmina, los cuales son utilizados para la síntesis de proteínas musculares y el mantenimiento de la masa muscular. Por otro lado, las bebidas vegetales presentaron un bajo aporte de proteína $(0.15$ a $0.35 \mathrm{~g} / 100$ $\mathrm{mL}$ ), excepto la bebida vegetal de soya cuyo contenido (3 g) es casi el mismo que la leche de vaca. ${ }^{5}$

De acuerdo con la NOM-155-SCFI-2012, todas las leches evaluadas cuentan con el mínimo de $3 \mathrm{~g} / 100$ $\mathrm{mL}$ de grasa butírica. ${ }^{2}$ El contenido de lípidos en estas leches está constituido por ácidos grasos saturados (butírico y caproico), y un alto porcentaje de triglicéridos, sin embargo también se incluyen vitaminas liposolubles, fosfolípidos como esfingomielina, la cual se ha demostrado tener un efecto protector frente al cáncer.

Mientras que por otro lado, las bebidas vegetales presentan un menor contenido de lípidos $(<1.6 \mathrm{~g} / 100 \mathrm{~mL})$, aunque con un buen perfil lipídico ya que provienen de cereales (arroz y avena) o de oleaginosas (almendra y nuez). Este perfil lipídico contribuye a tener un buen aporte de vitaminas del complejo $B$, ácidos grasos mono y poliinsaturados, lo cual puede contribuir con una disminución en las lipoproteínas de baja densidad LDL y coadyuvar a los procesos de oxidación e inflamación. ${ }^{7}$

Respecto a la concentración de vitaminas $A$ y $D$ no hubo diferencias en ambos tipos de producto. La vitamina $D$ se encuentra como vitamina D3 o colecalciferol, y como vitamina D2 o ergocalciferol que no es de origen animal ya que se utiliza para fortificar los alimentos. ${ }^{5,6}$

El calcio presente en ambos tipos de leche se encuentra en un rango de $100-171 \mathrm{mg} / \mathrm{mL}$ considerados como buenas fuentes cuando se encuentran en un rango de $120 \mathrm{mg} / 100 \mathrm{~mL} .{ }^{8}$ Sin embargo, se ha verificado que la biodisponibilidad de calcio es menor en las bebidas vegetales en comparación al proveniente de la leche de vaca, debido a que el calcio es añadido y no está asociado a las proteínas o a los folatos provenientes de los productos vegetales. ${ }^{9}$ Respecto al sodio en la leche de vaca como en la bebida vegetal comprenden un rango de 24.5-69.5 mg/100 mL, considerados como un bajo contenido de sodio. ${ }^{10}$

Una dieta adecuada debe aportar $25 \mathrm{~g} / \mathrm{dia}$ de fibra de diferentes fuentes. ${ }^{[11]}$ Uno de los componentes de dichas bebidas vegetales es la fibra, con valores de $0.1 \mathrm{a}$ $1.5 \mathrm{~g} / 100 \mathrm{~mL}$, cubriendo hasta entre 0.4 y $6 \%$ de las recomendaciones establecidas. Los beneficios de la presencia de fibra están relacionados con el buen funcionamiento del tracto gastrointestinal y una menor incidencia de enfermedades no transmisibles. De igual manera el contenido de fibra se ve modificado en las bebidas vegetales dependiendo el tipo de materia prima que se utiliza. ${ }^{12}$

La actualización de la NOM-051-SCFI/SSA12010 establece el contenido de nutrientes críticos e ingredientes que representan riesgos para la salud en alimentos procesados. Por lo que establece que el etiquetado frontal de estos productos deberá contar con sellos de advertencia, cuando se sobrepasan los contenidos nutrimentales de los criterios establecidos: las calorías tienen que ser $\leq$ a $275 \mathrm{kcal}$, azúcares $\leq$ al $10 \%$ total de energía y sodio $\leq 350 \mathrm{mg}$ establecidos en un contenido de $100 \mathrm{~mL}$. La leche de vaca y las bebidas vegetales se encuentran dentro de estos criterios establecidos. La única excepción fue la leche de soya, amaranto y coco, en donde sobrepasan los límites, al tener un aporte mayor al $10 \%$ de azúcares, por lo cual deben tener en su etiqueta sellos que adviertan un exceso de azúcares. ${ }^{10} \mathrm{El}$ consumo de azúcares está relacionado con problemas en torno a la obesidad y reducción de la sensibilidad en la insulina por lo cual el consumo de las bebidas vegetales debe ser moderado. ${ }^{5}$

\section{Conclusión}

Si bien las bebidas vegetales pueden ser utilizadas como una alternativa para sustituir la leche de vaca para grupos específicos de la población, es importante considerar que, aunque el aporte calórico es menor, la calidad de nutrientes no es la misma, debido al aporte de calcio, grasa butírica, lactosa y aminoácidos como la caseína y la lactoalbúmina presente en la leche de vaca. Además, algunas de las bebidas vegetales de acuerdo a la NOM051-SCFI/SSA1-2010 deben tener un etiquetado por exceso de azúcares, por lo que su consumo debe ser moderado.

\section{Referencias}

[1] Gómez DA, Mejía OB. Composición nutricional de la leche de ganado vacuno. Rev. Lasallista de Inv. 2005;2(1):38-42.

[2] NORMA Oficial Mexicana. NOM-155-SCFI-2012. LecheDenominaciones, especificaciones fisicoquímicas, información comercial y métodos de prueba. Diario Oficial de la Federación. [Internet]. 15 Marzo 2012. [consultado: 25 Agosto 2020]. Disponible en: http://www.dof.gob.mx/normasOficiales/4692/seeco/seeco.htm.

[3] Fuentes CA. Cambios en el consumo y percepciones en torno a la alimentación saludable de la leche tradicional y bebidas de origen vegetal. RIVAR (Santiago). 2019;6(17):1-14. 
Publicación semestral, Educación y Salud Boletín Científico Instituto de Ciencias de la Salud Universidad Autónoma del Estado de

Hidalgo, Vol. 9, No. 18 (2021) 122-125

[4] Jarasiunas E. Los productos puramente vegetales no pueden comercializarse con denominaciones tales como «leche», «nata», «mantequilla», «queso» o «yogur»: TJ, Séptima, 14 junio 2017. Asunto C-422/16: TofuTown. com. La Ley Unión Europea. 2017(51):12.

[5] García-Saavedra, NM. Bebidas vegetales (Tesis de pregrado). Madrid, España: Universidad complutense; 2017. Disponible en: https://eprints.ucm.es/56464/

[6] Fernández E, Martínez J, Martínez V, Moreno J, Collado L, Hernández M, Morán F. Documento de consenso: importancia nutricional y metabólica de la leche. Nutr Hosp. 2015;31(1):92-101.

[7] Griffiths MW. Improving the Safety and Quality of Milk: Canadá: Woodhead Publishing; 2010

[8] Farré R. La leche y los productos lácteos: fuentes dietéticas de calcio. Nutr Hosp. 2015; 31(2).

[9] Rodríguez HJ, Rodríguez LA, González AO, Dolores MM. Leche y productos lácteos como vehículos de calcio y vitamina D: papel de las leches enriquecidas. Nutr Hosp. 2019;36(4).

[10] Norma Oficial Mexicana. NOM-051-SCFI/SSA1-2010. Especificaciones generales de etiquetado para alimentos y bebidas no alcohólicas preenvasados-Información comercial y sanitaria. [Internet]. 5 de abril de 2010. [consultado: 12 de Septiembre 2020]. Disponible en: https://www.dof.gob.mx/2020/SEECO/NOM_051.pdf

[11]Secretaría de Salud. ¿Cuánta fibra dietética se debe consumir? [Internet]. 9 de julio de 2016. [Consultado 12 de Septiembre 2020]. Disponible en: https://www.gob.mx/salud/articulos/cuanta-fibradietetica-se-debeconsumir\#: : :text=Por $\% 20$ eso $\% 2 \mathrm{C} \% 20 \mathrm{el} \% 20 \mathrm{comit} \% \mathrm{C} 3 \% \mathrm{~A} 9 \% 20 \mathrm{de}, \mathrm{d}$ e\%20fibra\%20diet\%C3\%A9tica\%20al\%20d\%C3\%ADa.

[12] Escudero, AE. y González, SP. La fibra dietética. Nutr. Hosp. 2006; 21(2): 61-72. 